\title{
A Diffusion-Based Distributed Collaborative Energy Detection Algorithm for Spectrum Sensing in Cognitive Radio*
}

\author{
Junfang Li ${ }^{1}$, Wenxiao Chen ${ }^{2}$, Shaoli Kang ${ }^{3}$, Yongming Guo ${ }^{1}$ \\ ${ }^{1}$ The National Institute of Radio Spectrum Management, Xi'an, China \\ ${ }^{2}$ School of Communication and Information Engineering, Xi' an University of Posts \& Telecommunications, Xi'an, China \\ ${ }^{3}$ State Key Lab of Wireless Mobile Communication, China Academy of Telecommunication Technology, Beijing, China \\ Email:1i_jf@aliyun.com; chenwen13333@163.com; kangshaoli@catt.cn; ym_guo@126.com
}

Received July, 2013

\begin{abstract}
Spectrum sensing is one of the most important steps in cognitive radio. In this paper, a new fully-distributed collaborative energy detection algorithm based on diffusion cooperation scheme and consensus filtering theory is proposed, which doesn't need the center node to fuse the detection results of all users. The secondary users only exchange information with their neighbors to obtain the detection data, and then make the corresponding decisions independently according to the pre-defined threshold. Simulations show that the proposed algorithm is more superior to the existing centralized collaborative energy detection algorithm in terms of the detecting performance and robustness in the insecurity situation.
\end{abstract}

Keywords: Collaborative Energy Detection; Data Diffusion; Cognitive Radio; Spectrum Sensing

\section{Introduction}

Cognitive radio (CR) [1] is a spectrum sharing technology that allows unlicensed (secondary) users to operate in the licensed spectrum bands. It effectively improves the spectrum utilization in wireless communications [2].

Spectrum sensing is one of the key technologies of cognitive radio systems, and its purpose is to timely detect 'spectrum holes' and makes use of it. At present, the spectrum sensing algorithms mainly include matched filtering [3], cyclostationary feature detection [4] and energy detection [5]. The energy detection is the simplest method, which doesn't need the prior knowledge of primary user and is simple to implement [6].Since the detection problem such as 'hidden terminal' may be appeared relying on solely one terminal or node, the multi-node cooperative strategy in cognitive radio is researched and become one of the effective measures to solve the problem [7-11].

Based on whether depending on center node or not, multiple node energy detection algorithms can be divided into two kinds of cooperative models, that is, centralized and distributed. Currently, most collaborative spectrum

${ }^{*}$ This work is supported by National Science and Technology Major Project (2012ZX03003005-002). sensing algorithms can be viewed as centralized one. It means that center node (i.e. fusion center) can use the observed data or detection results of secondary users, and then decides whether the primary user is present. But there are some limits for the performance when the channels to the center node are under deep fading [10]. And this approach requires sufficient communications resources to transmit the data, which could easily lead to network congestion and increase the risk of the network collapse due to the presence of the central node [11]. However, in the distributed approach, where all nodes are in equal position and there is no center node, every node exchanges information only with its neighborhoods to achieve cooperative energy detection. [12] proposes a diffusion cooperation strategy based on peer-to-peer diffusion protocol, which attracts widespread concern due to its simple distributed architecture and low computational complexity.

In this paper, a distributed cooperative energy detection algorithm based on the diffusion strategy is proposed, in which the diffusion collaboration strategy is applied into spectrum sensing, and combines with consensus filtering theory to obtain a fully distributed energy detection algorithm. The proposed algorithm is simulated and compared with the existing collaborative algorithms. 


\section{Energy Detectioin and Network Topology}

\subsection{Energy Detection Model}

In this paper, we assume all users experience independent and identically distributed (iid) fading communication environment. Figure 1 depicts the block-diagram of an energy detector.

In the diagram, the received signal $y(t)$ is filtered by a bandpass filter with the center frequency $f_{s}$, and the bandwidth of interest $B$. This filter is followed by a squaring device $(\bullet)^{2}$ to measure the received energy and an integrator $\int_{0}^{T}$ is operated over the observation interval $T$. At last, the detection statistic of the integrator's output, $Y$, is compared with a threshold, $\lambda$, to decide whether primary user is present.

For implementation simplicity, the goal of spectrum sensing is viewed as two hypotheses test,

$$
\mathbf{y}(t)= \begin{cases}\mathbf{n}(t), & H_{0} \\ h \bullet \mathbf{s}(t)+\mathbf{n}(t), & H_{1}\end{cases}
$$

where $y(t)$ is the received signal by secondary user and $s(t)$ is transmitted signal of the primary user, $h$ is the channel amplitude and $n(t)$ is the additive white Gaussian noise (AWGN) with mean zero and variance $\sigma^{2}$. $H_{0}$ and $H_{1}$ represent the absence and the presence of the primary user, respectively. We also denote by $\gamma$ the signal-to-noise ratio (SNR). Correspondingly, the decision statistic $Y$ has the following form [5],

$$
Y \sim \begin{cases}\chi_{2 T B}^{2} & H_{0} \\ \chi_{2 T B}^{2}(2 \gamma) & H_{1}\end{cases}
$$

where $\chi_{2 T B}^{2}$ denotes the central chi-square distribution with $2 T B$ degrees of freedom, $\chi_{2 T B}^{2}(2 \gamma)$ denotes the non-central chi-square distribution with $2 T B$ degrees of freedom and the parameter of $2 \gamma$.

In the centralized collaborative scheme, center node uses the observed data or detection results of all secondary users to get the fusion result, and then compares with the pre-defined threshold $\lambda$ to make a final decision.

\subsection{The Diffusion-based Collaborative Distributed Network Model}

In the distributed energy detection scenario with diffusion collaborative strategy [12], the network formed by the secondary users can be represented by an undirected graph $G=(V, E)$ (Figure 2). The graph consists of a set of nodes $V=\{1,2, \cdots, n\}$ and a set of edges

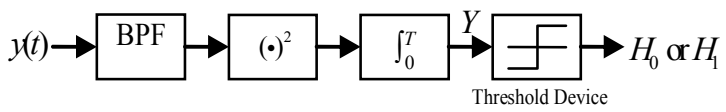

Figure 1. Block diagram of an energy detector.

$$
E=\{(i, j) \mid i, j \in V\} .
$$

If two secondary users are connected by an edge, it means that they satisfy $(i, j) \in E$ and can mutually exchange information. For convenience of description, we often refer node $i$ as $i$-th secondary user. Denote the neighbors of node $i$ by $N_{i}=\{j \in V \mid(i, j) \in E\} \in V$, and the number of elements in $N_{i}$ is denoted by $d_{i}$ (also called the degree of node $i$ ). E.g., the neighbor of node 1 is $N_{1}=\{1,2, n\}$ and $d_{1}=3$.

In the above distributed network, at time $k=0$, every user $i$ sets $x_{i}(k=0)=Y_{i}$ as its local state variable. The collaboration strategy of diffusion is represented by Figure 3, where every node $i$ in the network continuously combines the measurement results from its neighborhoods, and gains a fusion result. Those iterations are done repeatedly until a common result (i.e. steady state) is reached, $x^{*}=x_{i}(k)$, for any user $i$ at a certain time $k$. This process may be considered as the consensus filtering problem. Therefore, we can make use of consensus filter theory to attain collaborative spectrum sensing.

\subsection{Consensus Filtering Theory}

By achieving consensus, the individual variable $x_{i}$ progressively converges to the common value $x^{*}$, for each $i \in V$ i.e.

$$
x_{i}(k) \rightarrow x^{*}, \quad k \rightarrow \infty
$$

Our scheme is based on the recent results in weighting consensus algorithms [15], which the iterative formula is as follows:

$$
x_{i}(k+1)=w_{i i} x_{i}(k)+\sum_{j \in N_{i}} w_{i j} x_{j}(k)
$$

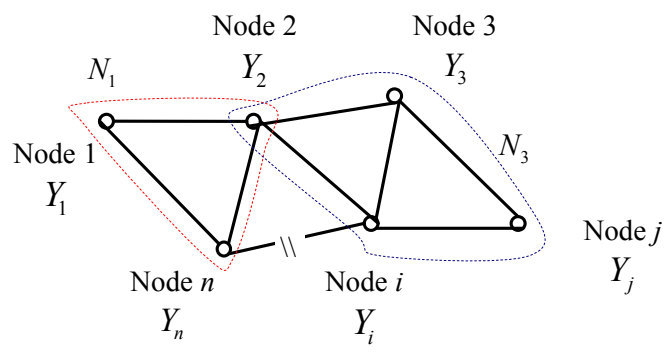

Figure 2. Distributed network with $n$ nodes.

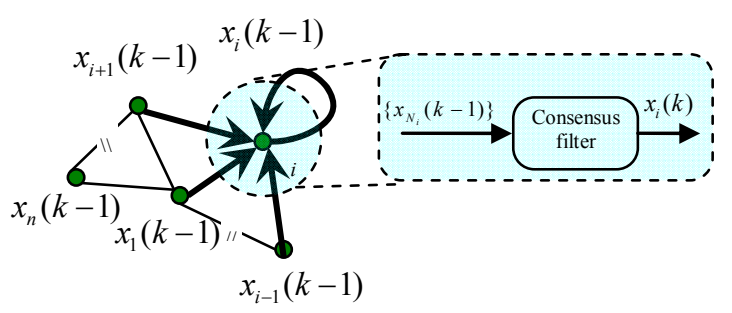

Figure 3. Network with a diffusion cooperation strategy. 
where, it is assumed that state variable $x_{i}(0)$ of the user $i$ is the measurement $Y_{i}$ at discrete time $k=0, w_{i i}$ and $w_{i j}$ are denoted the weighting factors. $w_{i j}=0$ means that nodes $i$ and $j$ are not connected. Currently, possible choices for the weighting coefficients $w$ are the Metropolis, the Laplacian and the nearest neighbor rules [16-18]. For simplicity, we choose to use the nearest neighbor rule which the combiner matrix $\boldsymbol{w}$ is defined as follows:

$$
w_{i j}= \begin{cases}\frac{1}{\left|N_{i}\right|}, & j \in N_{i} \\ 0, & \text { otherwise }\end{cases}
$$

According to the weighting factors, the diffusion cooperative scheme (4) can be interpreted as that every node $i$ exchanges information with its neighbors, and then updates itself measurement based on its own previous states and its neighbors.

\section{Diffusion-Based Algorithm for Spectrum Sensing}

When the network topology is setup, every node establishes communication links with its neighborhoods. The proposed diffusion-based collaborative spectrum sensing algorithm includes three stages, energy measurement, diffusion cooperative and decision.

1. Let $n$ denotes the number of users collaborating. Each node utilizes the spectrum sensing model (Figure 1) to make its measurements about primary users signal. $Y_{i}$ is denoted the measurement result of user $i$, and all $Y_{i}(i=1,2, \cdots, n)$ are random variables with independent and identical distribution by equation (2).

2. According to the network topology, every user uses the neighbors' measurements $Y_{j}, j \in N_{i}$ to cooperatively fuse. Those iterations are done repeatedly until a steady state is reached. Finally, we can obtain the decision statistic $T_{d}$ of the proposed algorithm which is the average measurements of all users, $\underline{\text { i.e. }}$. $T_{D}=\frac{1}{n} \sum_{i=1}^{n} Y_{i}$. We define $T_{M}=\sum_{i=1}^{n} Y_{i}$, therefore $T_{D}=\frac{1}{n} T_{M}$. According to the characteristic of the chi-square distribution, there is no primary signal present under $H_{0}$, we have

$$
\begin{aligned}
& T_{M} \sim \chi_{2 n T B}^{2} \\
& T_{D} \sim \frac{1}{n} \chi_{2 n T B}^{2}
\end{aligned}
$$

Under the hypothesis of $H_{1}$, all secondary users experience the same fading environment, and the detection threshold is determined only by the distribution of $T_{D}$ under $H_{0}$. So, there is no need to derive the distribution of $T_{D}$ under $H_{1}$.

3. Every secondary user compares the average measurement result with the pre-defined threshold $\lambda$ (Figure
1), and then makes a corresponding decision independently to get the final result of fusion locally,

$$
H= \begin{cases}H_{0}, & T_{D} \leq \lambda \\ H_{1}, & T_{D}>\lambda\end{cases}
$$

where the decision threshold $\lambda$ is determined by the equation $\int_{\lambda}^{+\infty} f_{T_{D}}\left(t_{d}\right) d t_{d}=P_{f}$ and the probability density function $f_{T_{D}}$ of the random variable $T_{D}$ can be derived from equation (7).

\section{Simulation Results and Discussions}

The performance of spectrum sensing algorithm is evaluated in terms of detection probability $P_{d}$ and false alarm probability $P_{f}$. As expected, the larger detection probability indicates that the performance of the algorithm is the better under a certain false alarm probability. In the simulation, we assume that $P_{f}=0.1$ and $T B=5$ at the selected center frequency $f_{s}$. There are 100000 times Monte Carlo simulation.

The detection performance of the proposed diffusionbased scheme for different number of collaborative spectrum sensors is shown in Figure 4. In the comparison, we can see that the performance of proposed scheme have a significant improvement with increasing number of collaborative spectrum sensors. For example, the detection probability of only one user is about $52 \%$ when SNR is $-5 \mathrm{~dB}$, while the number of the secondary users increases to 5 and 10 , the detection probability raises to about $67 \%$ and $75 \%$, respectively.

As to the network model with 10 users (Figure 2), Figure 5 shows the performance of the proposed diffusion-based scheme, the existing OR-rule and AND-rule collaborative scheme, respectively. From the simulation results, it can be observed that the proposed diffusionbased compares with the existing two kinds of algorithm

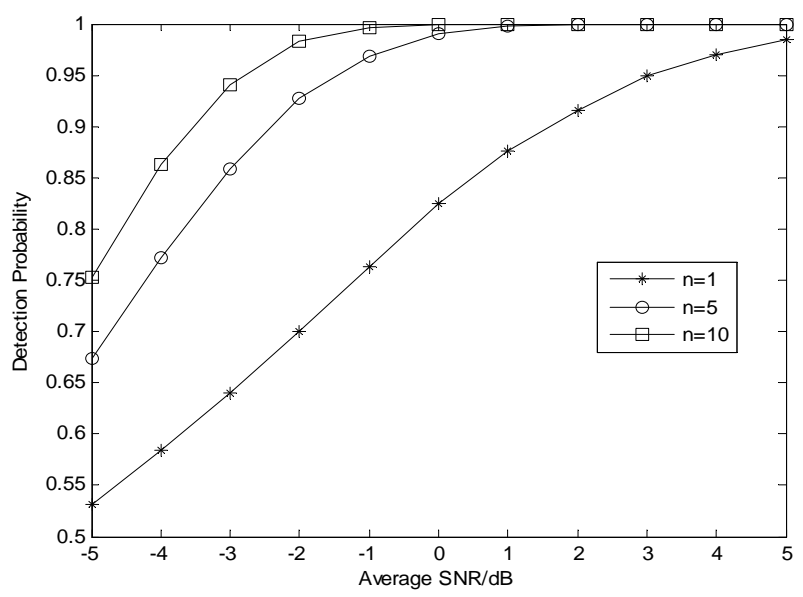

Figure 4. Detection performance comparison for different number of collaborative spectrum sensors. 


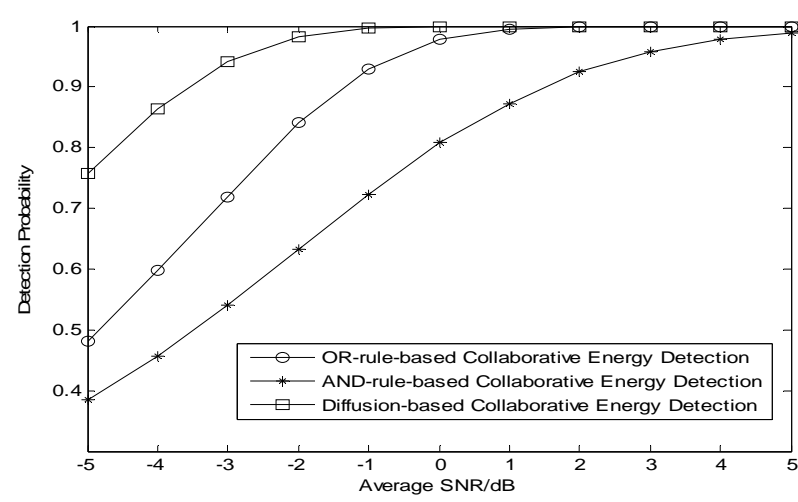

Figure 5. Detection performance comparison for $n=10$.

as the average SNR is $-4 \mathrm{~dB}$, the performance of detection probability improves to about $45 \%$ and $90 \%$, respectively.

\section{Conclusions}

In this paper, we have proposed a novel fully-distributed collaborative spectrum sensing algorithm in cognitive radios, in which every node exchanges information only with its neighbors and then makes the corresponding decision independently without fusion center. Simulation results demonstrate the validity of the proposed scheme, and show that it is more superior to the existing collaborative energy detection algorithms in terms of the detecting performance and robustness.

\section{REFERENCES}

[1] I. Mitola and J. G. Q Maguire, "Cognitive Radio: Making Software Radios More Personal," IEEE Personal Communications, Vol. 6, No. 4, 1999, pp. 13-18. doi:10.1109/98.788210

[2] J. Mitola, "Cognitive Radio: An Integrated Agent Architecture for Software Defined Radio," Doctor of Technology, Royal Inst. Technology (KTH), Stockholm, Sweden, 2000, pp. 271-350.

[3] D. Cabric, S. M. Mishra and R.W. Brodersen, "Implementation Issues in Spectrum Sensing for Cognitive Radios," Signals, Systems and Computers, 2004. Conference Record of the Thirty-eighth Asilomar Conference on. IEEE, 2004, pp. 772-776.

[4] C. Sun, W. Zhang and K. Ben, "Cluster-based Cooperative Spectrum Sensing in Cognitive Radio Systems," Communications, 2007. ICC'07. IEEE International Conference on, 2007, pp. 2511-2515.

[5] U. Harry. "Energy Detection of Unknown Deterministic Signals," Proceedings of the IEEE, Vol. 55, No. 4, 1967, pp. 523-531. doi:10.1109/PROC.1967.5573

[6] N. Hoven, R. Tandra and A. Sahai, "Some Fundamental Limits on Cognitive Radio," Wireless Foundations EECS, Univ. of California, Berkeley, 2005.
[7] Z. Quan, S. Cui, H. Poor and A. Sayed, "Collaborative Wideband Sensing for Cognitive Radios," Signal Processing Magazine, IEEE, Vol. 25, No. 6, 2008, pp. 60-73. doi:10.1109/MSP.2008.929296

[8] R. Chen, J. M. Park and K. Bian, "Robust Distributed Spectrum Sensing in Cognitive Radio Networks," The 27th Conference on Computer Communications. IEEE. 2008, pp. 1876-1884.

[9] S. M. Mishra, A. Sahai and R. W. Brodensen, "Cooperative Sensing Among Cognitive Radios," in Proc. IEEE International Conference on Communications (ICC), 2006, pp. 1658-1663.

[10] C. Sun, W. Zhang and K. Ben, "Cluster-based Cooperative Spectrum Sensing in Cognitive Radio Systems," Communications, 2007. ICC'07. IEEE International Conference on. IEEE, 2007, pp. 2511-2515.

[11] G.Y Lu, Y. X. Wang, K. Xie and X. N. Yong, "Novel Spectrum Sensing Method Based on the Spatial Spectrum for Cognitive Radio Systems," Journal of Electronics, Vol. 27, No. 5, 2010, pp. 625-629. doi:10.1007/s11767-011-0483-2

[12] C. G. Lopes and A. H. Sayed, "Diffusion Least-mean Squares Over Adaptive Networks: Formulation and Performance Analysis," Signal Processing, IEEE Transactions on, Vol. 56, No. 7, 2008, pp. 3122-3136. doi:10.1109/TSP.2008.917383

[13] F. F. Digham, M. S. Alouini and M. K. Simon, "On the Energy Detection of Unknown Signals over Fading Channels," Communications, IEEE Transactions on, Vol. 55, No. 1, 2007, pp. 21-24. doi:10.1109/TCOMM.2006.887483

[14] V. I. Kostylev, "Energy Detection of a Signal with Random Amplitude," Communications, 2002. ICC 2002. IEEE International Conference on. IEEE, Vol. 3, 2002, pp. 1606-1610.

[15] X. K. Wang, G. Y. Lu, Z. Q. Bao and H. Bai, "A Novel Distributed Cooperative Energy Detection Algorithm," Telecommunication Engineering, Vol. 52, No. 9, 2012, pp. 1480-1485.

[16] L. Xiao and S. Boyd, "Fast Linear Iterations for Distributed Averaging," Systems \& Control Letters, Vol. 53, No. 1, 2004, pp. 65-78. doi:10.1016/j.sysconle.2004.02.022

[17] R. S. Olfati and R. M. Murray, "Consensus Problems in Networks of Agents with Switching Topology and Time-delays," Automatic Control, IEEE Transactions on, Vol. 49, No. 9, 2004, pp. 1520-1533. doi:10.1109/TAC.2004.834113

[18] A. Jadbabaie, J. Lin and A. S. Morse, "Coordination of Groups of Mobile Autonomous Agents Using Nearest Neighbor Rules," Automatic Control, IEEE Transactions on, Vol. 48, No. 6, 2003, pp. 988-1001. doi:10.1109/TAC.2003.812781

[19] Z. Q. Li, F. Rchard and M. Y. Huang, "Distributed Spectrum Sensing in Cognitive Radio Networks," Wireless Communications and Networking Conference, IEEE, 5-8, 2009, pp. 1-5. 